\title{
II. InDEX OF PASSAgES
}

This Index is subdivided into four sections: A Greek Authors. B. Arabic Authors. C. Jewish Authors. D. European Authors. In each section, the authors are arranged in chronological order. For MSS. and editions used in connection with the works mentioned, see Bibliography II. MSS. are designated by asterisks.

\section{A. GREEK AUTHORS}

\begin{tabular}{|c|c|}
\hline Plato & Sophistic Elenchi \\
\hline & $2,165 \mathrm{a}, 38 \mathrm{ff} . \ldots$ \\
\hline 533D............ 568 & $2,165 b, 2 \ldots \ldots$ \\
\hline Timaeus & \\
\hline $37 \mathrm{D} \ldots \ldots \ldots \ldots \ldots \ldots 62$ & Physics \\
\hline Aristotle & $\mathrm{I}, 2-4 \ldots \ldots \ldots \ldots 569,570$ \\
\hline Categories & $\mathrm{I}, \quad 4 \ldots \ldots \ldots \ldots \ldots \ldots \ldots \ldots$ \\
\hline $6,4 b, 20-25 \ldots \ldots \ldots \ldots \ldots 419$ & $\mathrm{I}, \quad 4,187 \mathrm{a}, 28-29 \ldots \ldots \ldots 572$ \\
\hline $6,6 a, 17-18 \ldots \ldots \ldots \ldots \ldots 375$ & $187 a, 32-34 \ldots \ldots \ldots 572$ \\
\hline $8,9 a, 29 \ldots \ldots \ldots \ldots \ldots \ldots 688$ & $188 a, 6 \ldots \ldots \ldots 666$ \\
\hline $8,9 b, 36 \ldots \ldots \ldots \ldots \ldots 548$ & $5,188 a, 31-34 \ldots \ldots \ldots 698$ \\
\hline $8,10 a, 11-12 \ldots \ldots \ldots \ldots \ldots \ldots 67$ & $6,189 a, 34-189 b, 1 \ldots \ldots 698$ \\
\hline $8,10 a, 14-19 \ldots \ldots \ldots \ldots \ldots 688$ & $7 \ldots \ldots \ldots \ldots \ldots \ldots 71$ \\
\hline $8,10 \mathrm{a}, 16 \ldots \ldots \ldots \ldots \ldots \ldots 87$ & $7,190 \mathrm{a}, 31 \mathrm{ff} . \ldots \ldots \ldots 499$ \\
\hline $14,15 \mathrm{a}, 13 \mathrm{ff} . \ldots \ldots \ldots \ldots \ldots .499$ & 190b, 23-27.......700 \\
\hline & $1,192 b, 20-23 \ldots \ldots \ldots 672$ \\
\hline Prior Analytics & III, $1,200 b, 33-34 \ldots \ldots \ldots 499$ \\
\hline $\mathrm{I}, 31,46 \mathrm{a}, 31 \mathrm{ff} . \ldots \ldots \ldots \ldots . .332$ & $200 \mathrm{~b}, 34 \ldots \ldots \ldots \ldots 500$ \\
\hline$I, 32,47 a, 8 \ldots \ldots \ldots \ldots \ldots 456$ & $201 a, 10-11 \ldots \ldots \ldots 523$ \\
\hline $\mathrm{II}, 18,66 \mathrm{a}, 16 \ldots \ldots \ldots \ldots \ldots 472$ & 201a, 23-27......529 \\
\hline & $2,201 b, 20-22 \ldots \ldots \ldots 527$ \\
\hline Posterior Analytics & $201 \mathrm{~b}, 27 \mathrm{ff} \ldots \ldots \ldots 526$ \\
\hline $\mathrm{I}, \quad 4,73 \mathrm{a}, 21 \mathrm{ff} . \ldots \ldots \ldots$ & $201 b, 31 \ldots \ldots \ldots 526$ \\
\hline $\mathrm{I}, 10,76 \mathrm{~b}, 35 \mathrm{ff} \ldots \ldots \ldots \ldots \ldots 466$ & $202 a, 2-3 \ldots \ldots \ldots 466$ \\
\hline $\mathrm{I}, 24,85 \mathrm{a}, 13 \mathrm{ff} . \ldots \ldots \ldots \ldots \ldots 462$ & $202 a, 3-7 \ldots \ldots \ldots \ldots 562$ \\
\hline II, $4,91 \mathrm{a}, 16 \ldots \ldots \ldots \ldots \ldots 526$ & $202 a, 7 \ldots \ldots \ldots 526$ \\
\hline & $202 a, 7-8 \ldots \ldots \ldots \ldots 523$ \\
\hline Topics & $3,202 a, 21-31 \ldots \ldots \ldots .528$ \\
\hline II $, 8,113 b, 25-26 \ldots \ldots \ldots \ldots 541$ & $4 \ldots \ldots \ldots \ldots \ldots 330$ \\
\hline IV $, 1,120 b, 26-27 \ldots \ldots \ldots .506$ & $4-8 \ldots \ldots \ldots \ldots \ldots \ldots \ldots \ldots$ \\
\hline IV $, 1,121 \mathrm{a}, 30 \mathrm{ff} \ldots \ldots \ldots \ldots 499,549$ & $5,204 a, 8-14 \ldots \ldots \ldots 329$ \\
\hline$V I, 1,139 a, 31 \ldots \ldots \ldots \ldots \ldots 23$ & $204 a, 14-17 \ldots \ldots 330,334$ \\
\hline VI, $2,139 b, 19 f f \ldots \ldots \ldots \ldots \ldots 523$ & $204 a, 17-19 \ldots \ldots \ldots 330$ \\
\hline VI, $4,142 \mathrm{a}, 34 \mathrm{ff} . \ldots \ldots \ldots \ldots \ldots 523$ & $204 a, 20-32 \ldots \ldots \ldots 331$ \\
\hline
\end{tabular}




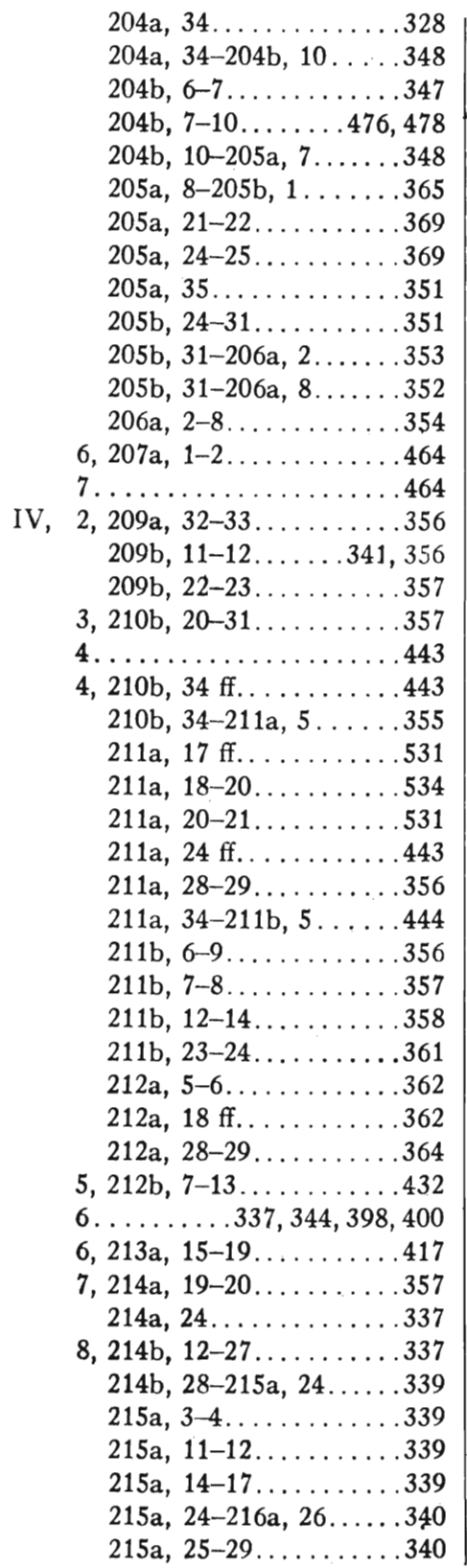

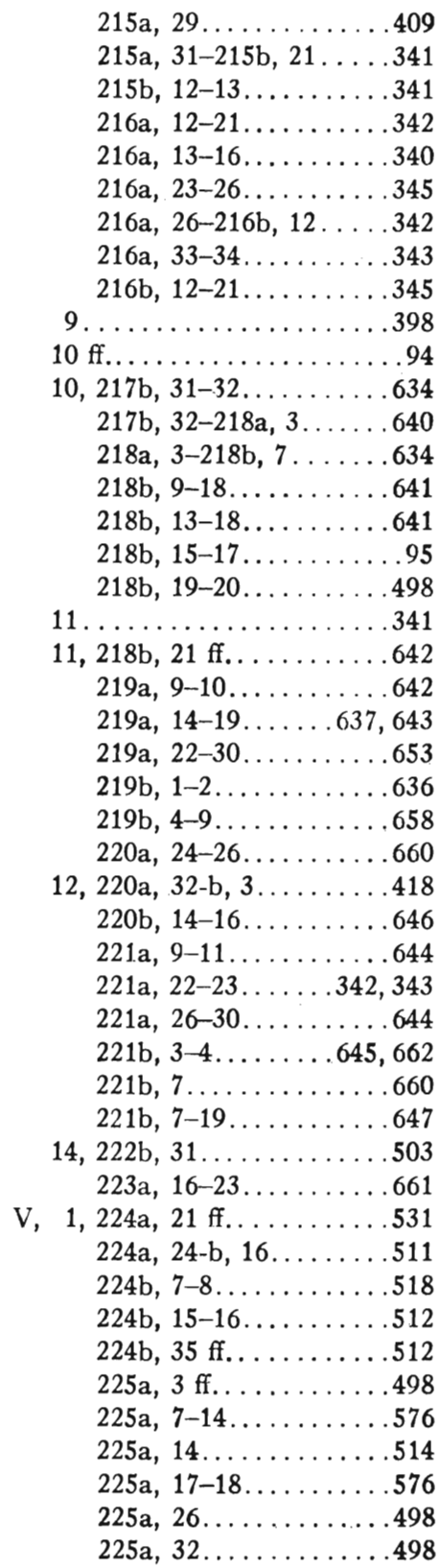




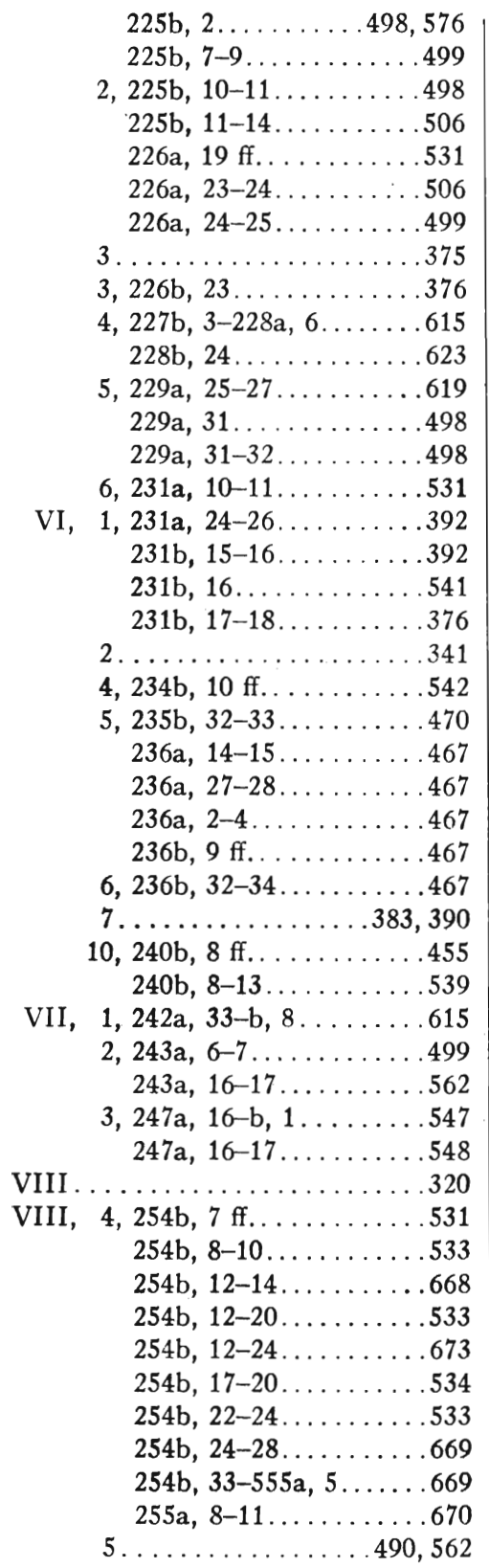

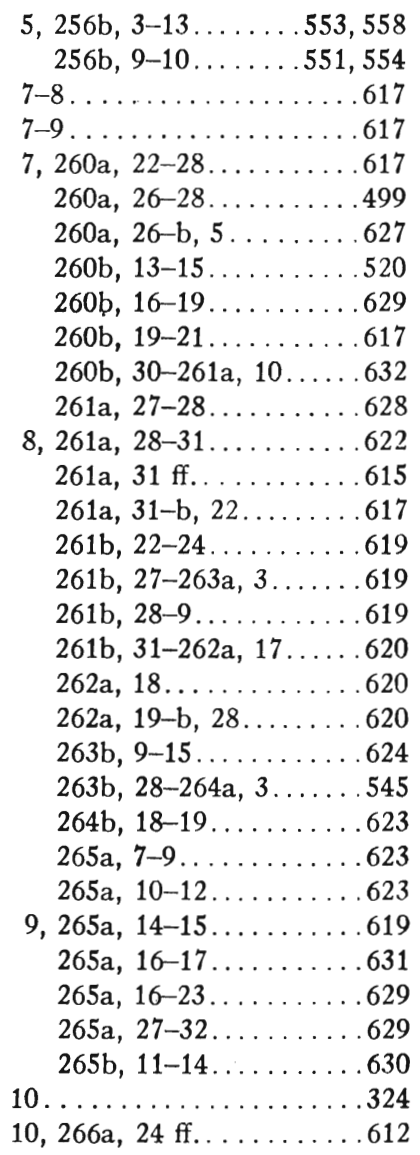

De Caelo

I, $1,268 \mathrm{a}, 6 \ldots \ldots \ldots \ldots . \ldots 54$ $268 \mathrm{a}, 7 \mathrm{ff} . \ldots \ldots \ldots . \ldots 541$

2, 268b, 17-18........619

269b, 6-9..... .553,558

$3 \ldots \ldots \ldots \ldots \ldots \ldots 429,431$

$3,270 \mathrm{~b}, 1-4 \ldots \ldots \ldots \ldots .614$

$5-7 \ldots \ldots \ldots \ldots \ldots 328,365$

$5,271 \mathrm{~b}, 4-6 \ldots \ldots \ldots \ldots . .397$

271b, $8-9 \ldots \ldots \ldots \ldots 472$

271b, 27-272a, $7 \ldots \ldots . .379$

$272 a, 7-20 \ldots \ldots \ldots \ldots 382$

$272 \mathrm{a}, 7-11 \ldots \ldots \ldots . .383$ 


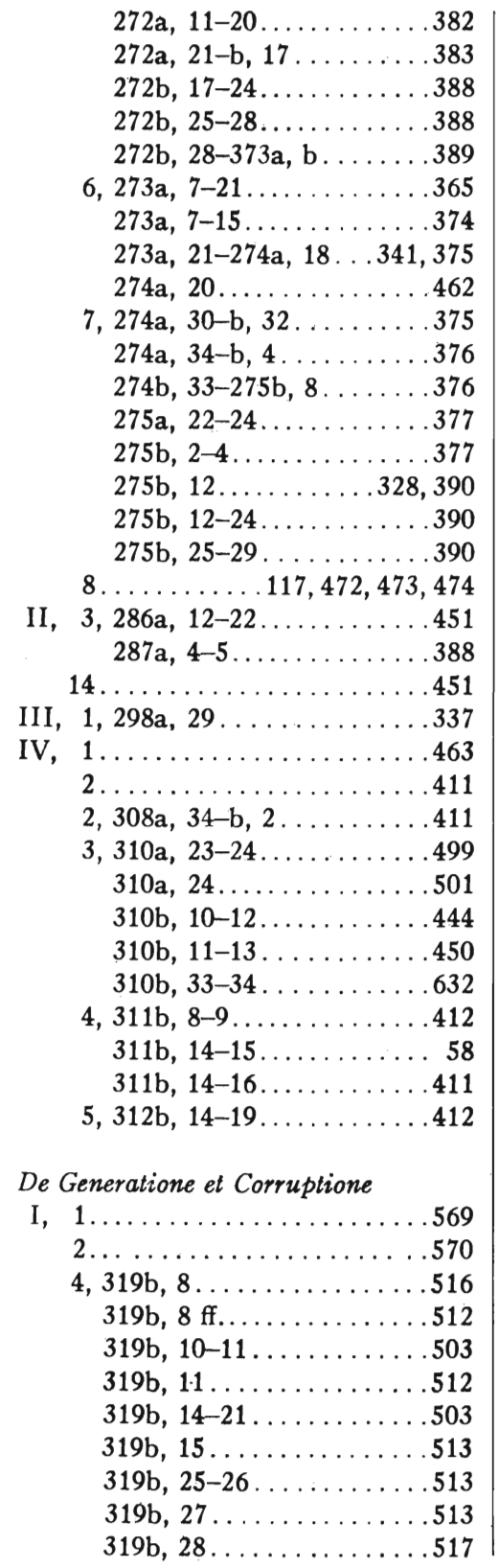

$319 \mathrm{~b}, 29 \ldots \ldots \ldots \ldots \ldots 513$

$319 \mathrm{~b}, 30 \ldots \ldots \ldots \ldots . \ldots 513$

$319 \mathrm{~b}, 31 \mathrm{ff} \ldots \ldots . .499,503,450$

319b, 31-320a, 2 . .....512 $319 \mathrm{~b}, 33 \ldots \ldots .501,513,516$ $320 \mathrm{a}, 1 \ldots \ldots \ldots .513,516$ $320 \mathrm{a}, 2 \ldots \ldots \ldots \ldots \ldots 513$ $320 \mathrm{a}, 17-19 \ldots \ldots \ldots \ldots .520$

$320 \mathrm{a}, 19-22 \ldots \ldots \ldots \ldots 521$

$5,322 \mathrm{a}, 16 \ldots \ldots \ldots \ldots .633$

II, $1-4 \ldots \ldots \ldots \ldots \ldots \ldots . \ldots 573$

1, 329a, 24-26.........591

$4,331 \mathrm{a}, 11 \ldots \ldots \ldots \ldots \ldots 444$

$331 \mathrm{a}, 13 \mathrm{ff} . \ldots \ldots \ldots \ldots . . .450$

De Anima

I, 1, 403b, 12-15 ..........392

$2,405 \mathrm{a}, 19-21 \ldots \ldots \ldots \ldots 568$

$3,406 \mathrm{a}, 4 \mathrm{ff} . \ldots \ldots \ldots \ldots . \ldots 531$

$406 \mathrm{a}, 12 \mathrm{ff} . \ldots \ldots \ldots . .499$

$4,407 \mathrm{~b}, 31 \ldots \ldots \ldots \ldots .560$

$408 \mathrm{a}, 14 \ldots \ldots \ldots \ldots 560$

$408 \mathrm{~b}, 2-4 \ldots \ldots \ldots 548,549$

$408 \mathrm{~b}, 4 \ldots \ldots \ldots \ldots \ldots 54$

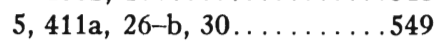

II, 1, 212a, 22-27........ 525

$2,414 a, 19-22 \ldots \ldots \ldots \ldots 560$

$7,419 a, 15-21 \ldots \ldots \ldots .471$

III, $3,427 \mathrm{~b}, 14-16 \ldots \ldots \ldots . .546$

$9,432 \mathrm{a}, 15-17 \ldots \ldots \ldots \ldots 607$

De Sensu

$2,438 \mathrm{a}, 27 \ldots \ldots \ldots \ldots 471$

6, 446b, 29-447a, 2......464

De Motu Animalium

$1,698 \mathrm{a}, 15-\mathrm{b}, 1 \ldots \ldots \ldots .451$

Metaphysics

I, 3, 984a, 21-25......700

II, $2 \ldots \ldots \ldots \ldots \ldots \ldots 490$

2, 994a, 1 ff. .....482, 495

994a, $11-15 \ldots \ldots \ldots 482$

994a, $15-16 \ldots \ldots \ldots 482$

994a, $16-19 \ldots \ldots \ldots 483$

994a, $18-19 \ldots \ldots .492$

$494 \mathrm{~b}, 9 \ldots \ldots \ldots . \ldots 359$ 
$\mathrm{V}, \quad 4,1015 \mathrm{a}, 10-11 \ldots \ldots . .359$

$5,1015 a, 33-34 \ldots \ldots \ldots 109$

$8 \ldots \ldots \ldots \ldots \ldots \ldots 53$

$8,1017 \mathrm{~b}, 13-14 \ldots \ldots \ldots 573$

$1017 \mathrm{~b}, 25-26 \ldots .359,574$

$12,1020 \mathrm{a}, 5-6 \ldots \ldots \ldots \ldots 110$

$13,1020 \mathrm{a}, 7 \ldots \ldots \ldots \ldots .541$

$1020 \mathrm{a}, 8-11 \ldots \ldots \ldots 419$

$17 \ldots \ldots \ldots \ldots \ldots \ldots \ldots . \ldots \ldots$

$17,1022 a, 4-6 \ldots \ldots \ldots \ldots 357$

$19 \ldots \ldots \ldots \ldots \ldots . \ldots 60$

$19,1022 b, 1-3 \ldots \ldots \ldots \ldots 688$

$1022 b, 15-18 \ldots \ldots \ldots 688$

$22,1022 b, 22-31 \ldots \ldots \ldots 683$

$1022 \mathrm{~b}, 31-32 \ldots \ldots .683$

VI, 2, 1026b, 27-29.......681

VII, 3, 1029a, 1-3........575

1029a, 10-12......553

$4,1029 \mathrm{~b}, 13 \ldots \ldots \ldots . .328$

$7,1032 \mathrm{a}, 13-15 \ldots \ldots .499$

1032b, 1-2....... 359

1033a, $7-12 \ldots \ldots \ldots 512$

$10 \ldots \ldots \ldots \ldots \ldots \ldots \ldots 421$

$10,1035 a, 2 \ldots \ldots \ldots \ldots .575$

$1035 a, 17-21 \ldots \ldots \ldots 393$

11, 1036a, 28-29........359

$1037 \mathrm{a}, 27 \ldots \ldots \ldots 35$

$13,1038 b, 9-10 \ldots \ldots \ldots .699$

VIII, 1, 1042a, $26 \mathrm{ff} . \ldots \ldots \ldots .575$

1042a, 27-28......556

1042a, 27-29.......686

1042a, 32-34.......5.573

1042a, 32-b, 3 . . 499, 520

1042b, 1-3.........546

1042b, 3-5 ... . . 519, 520

IX, $1,1046 a, 34-35 \ldots \ldots \ldots 683$

$4,1046 \mathrm{~b}, 12-14 \ldots \ldots \ldots 343$

1047b, 4-5 .........551

$6,1048 a, 30-35 \ldots \ldots \ldots .691$

$7 \ldots \ldots \ldots \ldots \ldots 62$

7, 1048b, 37-1049a, 5 . . 692

$1049 a, 5-18 \ldots \ldots \ldots .692$

$8,1049 \mathrm{~b}, 35 \mathrm{ff} . \ldots \ldots \ldots 467$

1050b, 11-12 ......551

$\mathrm{X}, \quad 4,1055 \mathrm{a}, 5 \ldots \ldots \ldots \ldots 375$

$\mathrm{XI}, 3,1061 \mathrm{a}, 19 \ldots \ldots . .52,464$
$9,1065 b, 16 \ldots \ldots \ldots \ldots 523$

1065b, 22-23... 523, 526

$10 \ldots \ldots \ldots \ldots \ldots \ldots \ldots 328$

10, 1066b, 1-7..........329

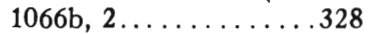

1066b, 7-9........ 330

1066b, 9-11.....331, 334

1066b, 11-21........ 332

1066b, 21-26........ 348

1066b, 22-1067a, 7....348

1066b, 24.......... 347

1066b, 24-26......476

1067a, 7-25........365

$1067 \mathrm{a}, 20 \ldots \ldots \ldots 369$

$1067 \mathrm{a}, 23-29 \ldots \ldots . \ldots 351$

1007a, 28-30........ 353

$1067 a, 28-33 \ldots \ldots \ldots 352$

1067a, 30-33 ....... 354

XII, 1, 1069a, 24........666

1069b, 3-9.........699

XII, 2-4 ............569

2, 1069b, 9 ff. . . . . . . 499

1069b, 24-26........ 596

1069b, 28-29.........692

$1069 \mathrm{~b}, 32-34 \ldots \ldots \ldots 572$

3, 1069b, 35-1070a, 2...700

$1070 \mathrm{a}, 9 \mathrm{ff} . \ldots \ldots \ldots .575$

4, 1070b, 13-14......575

$6,1071 b, 28-30 \ldots \ldots \ldots 700$

$7 \ldots \ldots \ldots \ldots \ldots \ldots \ldots 624$

$8,1074 a, 33-34 \ldots \ldots \ldots 666$

\section{Rhetoric}

I, 2, 1355b, 26-27........397

\section{Euclid}

\section{Elements}

I, Def. iii . . . . . . . . . . 392

I, Def. xiv.......... 388, 689

I, Def. xxiii . . . . . . . . . . 465

I, Post. i. . . . . . . . . 466

V, Def. i..............419

V, Def. ii..........4418,419

V, Def. v.............419

VII, Defs. viii-ix. . . . . . . 478

Arabic translation........... 339 


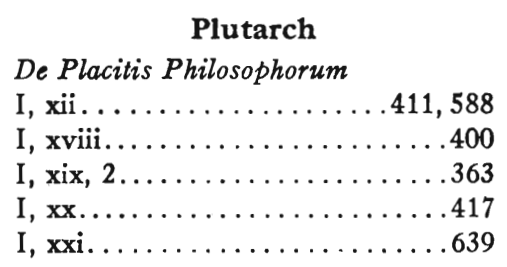

Enneads

II, iv, $1 \ldots \ldots \ldots \ldots \ldots \ldots \ldots 58$

II, iv, $8 \ldots \ldots \ldots \ldots \ldots 544,582$

II, iv, $9 \ldots \ldots \ldots \ldots \ldots .578,582$

III, vii, Introduction . . . . . . . 662

III, vii, $4 \ldots \ldots \ldots \ldots \ldots . \ldots 51,662$

III, vii, $6 \ldots \ldots \ldots \ldots \ldots 635,640$

III, vii, $6-9 \ldots \ldots \ldots \ldots \ldots \ldots 654$

III, vii, $10 \ldots \ldots \ldots \ldots \ldots \ldots 64$

III, vii, $11 \ldots \ldots \ldots \ldots \ldots \ldots 655$

III, vii, $12 \ldots \ldots \ldots \ldots \ldots \ldots 55$

\section{Themistius}

\section{In Physica}

IV, 5, p. $120 \ldots \ldots \ldots \ldots \ldots \ldots 437$

VI, 6, p. $197 \ldots \ldots \ldots \ldots \ldots \ldots 544$

In De Caelo (Hebrew)

I, (3), p. 9, 11. 26-27 ......597

I, (5), p. 14, ll. 19-21....... 397

I, (5), p. 14, ll. 24-26......472

I, 7, p. $27,11.10-17 \ldots \ldots \ldots 378$

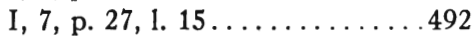

II, 3, p. 88, 1. 9......... 396

II, 3 , p. $91,1.31 \ldots \ldots \ldots \ldots . .396$
In De Caelo (Latin)

I, 3, p. 14, 11. 13-14.......597

I, 5, p. 22, 11. 4-7 ........ 397

I, 5, p. 22, ll. 13-15.......472

$\mathrm{I}, 7$, p. $40,1.35 \mathrm{ff} \ldots \ldots \ldots \ldots . \ldots 378$

I, 7, p. $41,1.4 \ldots \ldots \ldots \ldots \ldots 492$

II, 3, p. 131, 1l. 23-24....... . 396

II, 3 , p. 136, 1l. 33-34 ........ 396

\section{Simplicius}

In Physica

I, 7, pp. 227-233......581

I, 7, p. 227, 11. 26-30 . . .582

I, 7, p. $230,1.14 \ldots \ldots \ldots 582$

I, 7, p. 230, 11. 21-29. .578, 582

III, 1, p. $414,1.15$ ff. . . . . 526

IV, 1, p. $539,1.8 \mathrm{ff} . \ldots \ldots 356$

IV, 4, p. $571,1.25 \ldots \ldots \ldots 357$

IV, 4, p. 585, 1. 34 ff. . . . . 445

IV, 8, p. 665, 11. 9-10. . . . 401

IV, 8, p. 681, 11. 21-26. . . 4415

IV, 9, p. $693,1.11 \mathrm{ff} . \ldots \ldots .472$

IV, 10, p. 700, 11. 16-22. . . 635

IV, 12, p. 741, 11. 19-26. . . 646

VII, 3, p. 1075, 1. 23 ff. ... . . .547

VIII, 6, p. 1261, 11. 14-19. . .. 557

VIII, 6, p. 1261, 11. 19-21 . . . .559

In De Caelo

II, 3, p. 398, 11. 20-24 . . . . . . 455

\section{Lucretius}

De Rerum Natura

VI, 11, 998-1041...........563

\section{B. ARABIC AUTHORS}

Alkindi

Liber de Quinque Essentiis

p. 35 . .500

\section{Pseudo-Aristotle}

Theology of Aristotle

p. 107 . .662

p. 108 . .497

\section{Ihwan al-Safa}

Encyclopedia......362, 418, 421, 500, $578,580,635,655$

\section{Alfarabi}

Mahut ha-Nefesh. .586

\section{Avicenna}

Al-Najah:

Physics

p. $25 \ldots \ldots \ldots \ldots \ldots \ldots 673$

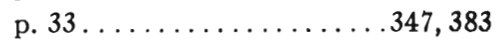

p. $34 \ldots \ldots \ldots \ldots \ldots \ldots \ldots . \ldots \ldots 6$

p. $41 \ldots \ldots \ldots \ldots \ldots \ldots \ldots 12$ 


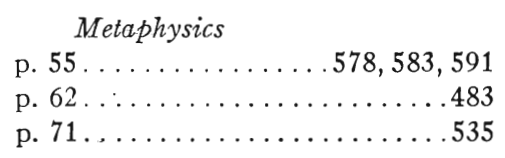

Al-Shafa................583

Treatise on Psychology........339

Commentary on De Caelo

IV. .594

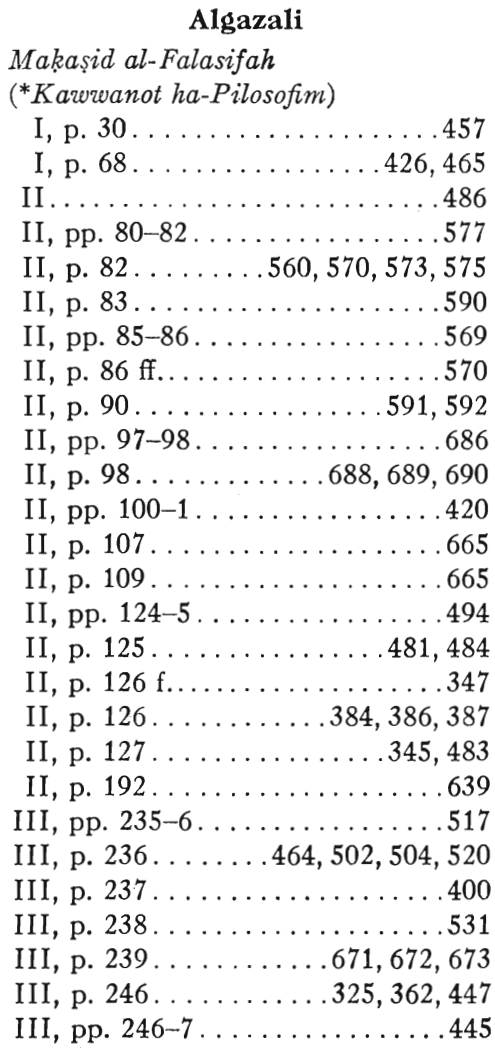

\section{Tahafut al-Falasifah}

( ${ }^{*}$ Happalat ha-Pilosofim)

I......369, $477,478,485,486,489$
IV $\ldots \ldots \ldots \ldots .483,485,493,595$

XIV 536

Mizan al-'Amal

Intr. p. $3 \ldots \ldots \ldots \ldots \ldots 326$

XXVII, p. $159 \ldots \ldots \ldots \ldots \ldots . . . \ldots 397$

XXVII, p. $162 \ldots \ldots \ldots \ldots . . . .397$

Mozene Zedek

Intr. pp. 6-7 . . . . . . . . . . 326

XXVII, p. $170 \ldots \ldots \ldots \ldots . .397$

XXVII, p. $172 \ldots \ldots \ldots \ldots \ldots 397$

Teshubot She'elot

p. XXXIX ...............496

pp. XLVIII-XLIX . . . . . . . 666

pp. LI-LII .............. 483

\section{Shahrastani}

Kitab al-Milal wal-Nihal

p. $253 \ldots \ldots \ldots \ldots \ldots \ldots \ldots . \ldots . \ldots . \ldots 21$

p. $311 \ldots \ldots \ldots \ldots \ldots \ldots \ldots . \ldots . \ldots . \ldots 21$

p. $312 \ldots \ldots \ldots \ldots \ldots \ldots . \ldots 21,325$

pp. $343-4 \ldots \ldots \ldots \ldots \ldots \ldots \ldots \ldots \ldots \ldots$

p. $345 \ldots \ldots \ldots \ldots \ldots \ldots \ldots \ldots \ldots \ldots \ldots$

p. $349 \ldots \ldots \ldots \ldots \ldots \ldots \ldots \ldots \ldots \ldots$

p. $357 \ldots \ldots \ldots \ldots \ldots \ldots \ldots . \ldots . \ldots . \ldots 37$

pp. $364-5 \ldots \ldots \ldots \ldots \ldots \ldots . \ldots 57$

p. $366 \ldots \ldots \ldots \ldots \ldots 578,583,591$

p. $397 \ldots \ldots \ldots \ldots \ldots \ldots . \ldots 2,503$

p. $398 \ldots \ldots \ldots \ldots \ldots \ldots 505,507$

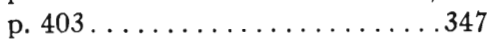

pp. $403-4 \ldots \ldots \ldots \ldots \ldots . .486$

\section{Averroes}

${ }^{*}$ Intermediate Commentary on the Categories

II, $2 \ldots \ldots \ldots \ldots \ldots \ldots \ldots . . .419$

II, iv, $5 \ldots \ldots \ldots \ldots \ldots \ldots 68$

Epitome of the Sophistic Elenchi

p. 55 a ................... 415

${ }^{*}$ Epitome of the Topics

p. $58 \mathrm{a}$. .342 


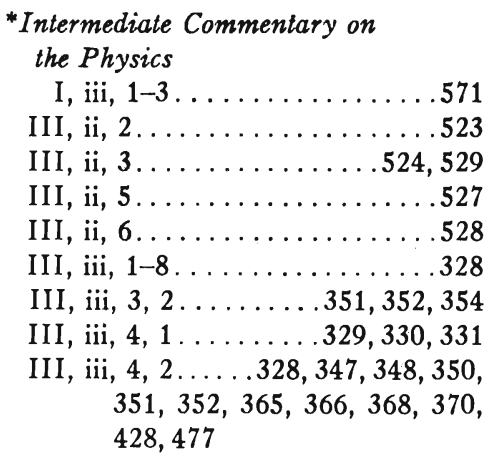

IV, $\mathrm{i}, 1,6 \ldots \ldots \ldots \ldots \ldots 355$

IV, $i, 1,8 \ldots \ldots 356,357,358$, $360,361,362$

IV $, i, 1,9 \ldots 327,433,451,453,455$

IV $, i, 1,10 \ldots \ldots \ldots \ldots \ldots 45$

IV, ii . . . . . . . . . . . . 357

IV, ii, $2 \ldots \ldots \ldots \ldots \ldots 398,399$

IV, ii, $5 \ldots \ldots 337,338,339,340$, $342,343,344,403$

IV, ii, 6........... 344

IV, iii............6.633

IV, iii, i.634, 637, 640, 642, 643, 644

IV, iii, $3 \ldots \ldots 6634,636,644$

IV, iii, $5 \ldots \ldots \ldots 645,646,647$

IV, iii, 7 . . ........661

V, ii, $3 \ldots \ldots \ldots 514,518,576$

V, ii, $4 \ldots \ldots \ldots \ldots .507,518$

V, iv, $1-2 \ldots \ldots \ldots \ldots \ldots 615$

VI, $7 \ldots \ldots \ldots 325,541,542,543$, $544,545,549$

VI, $12 \ldots \ldots \ldots \ldots \ldots \ldots . \ldots 539$

VII, 1...........669,670

VII, $3 \ldots \ldots \ldots \ldots \ldots \ldots 562,563$

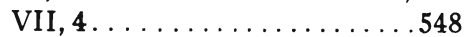

VIII, ii, $2 \ldots \ldots \ldots \ldots \ldots .490,495$

VIII, iv, 2 . . . . . . . . . . 669

VIII, iv, 4, $1 \ldots \ldots \ldots 668,673,674$

VIII, iv, 4, 2........554, 669

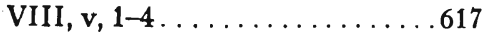

VIII, v, 2 . . . . . . . . . 618, 619

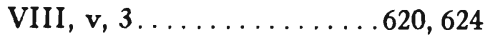

VIII, v, $4 \ldots \ldots 6623,628,629,630$

VIII, vi . . . . . . . . . . . 594

VIII, vi, $2 \ldots \ldots \ldots \ldots \ldots \ldots 612$
Epitome of the Physics

III, p. 10b. . . . . . 392, 429, 477

III, p. 11a . . . . . . . . . . 369

III, p. 11b. . . . . 332, 369, 374

III, pp. 12-13 ......... 464

IV, p. 13b........... 357

IV, p. $14 a \ldots \ldots \ldots \ldots \ldots \ldots 444$

IV, pp. 14b-15a........ 415

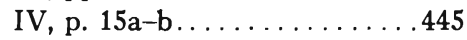

IV, p. 16b............ 439

IV, p. 17b...........6638

IV, p. $18 \mathrm{a} \ldots \ldots \ldots \ldots \ldots \ldots 636$

V, p. 21b.............544

V, p. 22a............... 399

VI, p. $25 b \ldots \ldots \ldots \ldots \ldots \ldots 376$

VI, p. $30 \mathrm{ff} . \ldots \ldots \ldots \ldots \ldots \ldots 54$

VI, p. $32 \mathrm{a} \ldots \ldots \ldots \ldots \ldots \ldots 470$

VII, p. 37a ........ 562, 566

VIII, p. 46b............. 492

*Long Commentary on the Physics

III, iii, $4,2 \ldots \ldots \ldots \ldots 347,477$

IV $, i, 1,9 \ldots \ldots \ldots \ldots \ldots .437$

IV, $i, 3 \ldots \ldots \ldots \ldots \ldots \ldots 11$

VI, iii, $1 \ldots \ldots \ldots$ 541, 542, 543

VIII, ii, $3 \ldots \ldots \ldots \ldots \ldots \ldots . .554$

VIII, iii, $1 \ldots \ldots \ldots \ldots \ldots \ldots 617$

VIII, iii, 2............6616

*Intermediate Commentary

on De Caelo

I, iv...................553

I, v-vi............. 614

I, vii . . . . 328, 365, 374, 375, 376, $378,379,380,382,383,384,385$, $388,389,390,472$

I, $x, 2,8 \ldots \ldots \ldots \ldots 59,613,681$

*Intermediate Commentary on De Generatione et Corruptione

I, $\mathrm{i}, 4 \ldots \ldots \ldots \ldots \ldots \ldots \ldots .504$

*Intermediate Commentary on the Meteorologica

I....................401

*Epitome of the Meteorologica

I...................444 


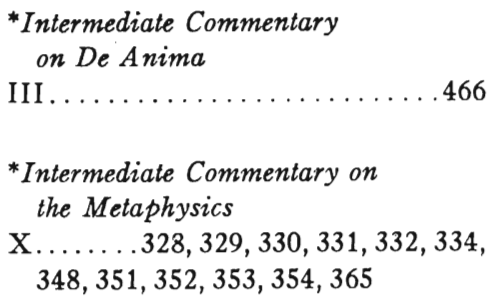

Epitome of the Metaphysics

II . . . . . . . . . . . . 579, 589

III ............... 482

*Sermo de Substantia Orbis. . . . . . 586, $594,612,614$

Tahafut al-Tahafut

( ${ }^{*}$ Happalat ha-Happalah)

I. . . . . . . . . . 327, 697

I, p. $7 \ldots \ldots \ldots \ldots \ldots \ldots 494$

I, p. $9 \ldots \ldots \ldots \ldots \ldots \ldots 489$

I, p. $10 \ldots \ldots \ldots \ldots \ldots 486$

III ............. . 327, 597

IV .................595

IV, p. $70 \ldots \ldots \ldots \ldots \ldots \ldots 495$

IV, p. $71 \ldots \ldots \ldots \ldots \ldots \ldots 47$

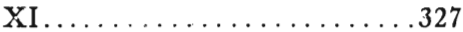

XIV................536
Philosophy and Theology

Arabic text, p. $66 \ldots \ldots \ldots 364,422$

\section{Altabrizi}

Commentary on Maimonides'

Twenty-five Propositions

Introduction . . . . . . . . . 19, 20

Prop. I . . . . . . 20, 346, 381, 382, 391

Prop. II . . . . . . . . . . . . . . 476

Prop. III . . . . 480, 481, 483, 484, 497

Prop. IV . . . . 399, 400, 503, 504, 521

Prop. V............ 522, 525

Prop. VI. . . . . . . . . . 21, 532, 534

Prop. VII . . . . . . 540, 546, 547, 549

Prop. VIII . . . . . . . . . . 552, 555

Prop. IX........... 562, 567

Prop. X............577, 578

Prop. XI .........602, 604, 613

Prop. XII ..............613

Prop. XIII . . . . . . . . . . . . 616

Prop. XIV . . . . . . . 399, 630, 631

Prop. XV. 633, 634, 635, 637, 638, 656

Prop. XVI . . . . . . . . . . . 665

Prop. XVII . . . . . 21, 668, 670, 674

Prop. XVIII . . . . 676, 677, 678, 679

Prop. XIX. . . . . . . . 682, 683

Prop. XX........... . 684, 685

Prop. XXI . . . . . . . . . . . . . 685

Prop. XXII . . 570, 585, 592, 687, 689

Prop. XXIII . . . . . . 690, 693, 696

\section{JEWISH AUTHORS}

\section{Bible, Talmud and Midrash}

Exodus 24, 16. . . . . . 459, 460

Isaiah $6,3 \ldots \ldots \ldots \ldots \ldots \ldots 48$

Psalms 19, 2........... 125

Job $16,19 \ldots \ldots \ldots \ldots \ldots \ldots \ldots 1$

Job $23,3 \ldots \ldots \ldots \ldots \ldots \ldots \ldots 417$

Ecclesiastes I, 14..........476

Ecclesiastes 6, 11......... . 474

Matthew 17, 20........... 342

Mishnah Ta'anit II, $1 \ldots \ldots \ldots 320$

Mekilta, Ki Tissa I...........458

Hagigah 11b...............476

Megillah 19b............570

Nedarim 25a . . . . . . . . . . 458
Sanhedrin 107b............562

Shebu'ot $7 \mathrm{~b} \ldots \ldots \ldots \ldots \ldots \ldots 458$

Shebu'ot $29 a \ldots \ldots \ldots \ldots \ldots \ldots 458$

Shebu'ot 39a............458

'Abodah Zarah 40b..........458

Horayot 11b. . . . . . . . . . .458

Genesis Rabbah 68, 9......458

Sefer ha-Bahir $48 \ldots \ldots \ldots \ldots \ldots 460$

\section{Anonymous}

* Commentary on Intermediate

Physics. (MS. Adler 1744.1)

III, ii, $3 \ldots \ldots \ldots \ldots \ldots 524,525$ 
* Commentary on Intermediate

Physics (MS. Adler 1744.2)

I, ii, 2, 4 .

$\mathrm{V}, \mathrm{ii}, 4$

511

* Commentary on Epitome of the

Physics (MS. Bodleian 1387)

III . . . . . . . . . . . . . . . . . . 373

\section{Philo}

De Somniis

I, 11 123

Fragments

Exodus 24, 16 .460

Sefer ha-Yesodot

\section{Isaac Israeli}

I, p. 11

I, p. 12 .574

II, p. 45 .392

III, pp. $62-63 \ldots \ldots \ldots \ldots \ldots .500$

III, p. $63 \ldots \ldots \ldots \ldots \ldots \ldots 501$

III, p. $71 \ldots \ldots \ldots \ldots \ldots \ldots 00$

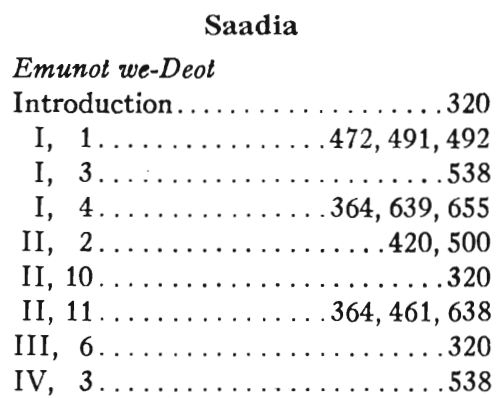

Commentary on Sefer Yeẓirah

p. $18 \ldots \ldots \ldots \ldots \ldots \ldots \ldots 420$

p. $72 \ldots \ldots \ldots \ldots \ldots \ldots \ldots 1$

\section{Samuel ha-Nagid}

Ben Kohelet. 491

\section{Ibn Janah}

Sefer ha-Shorashim $.335,563$

\section{Solomon Ibn Gabirol}

Likkutim min Sefer Mekor Hayyim

I, $6 \ldots \ldots \ldots \ldots \ldots \ldots 59,601$

II, $1 \ldots \ldots \ldots \ldots \ldots \ldots \ldots .579$

II, $2 \ldots \ldots \ldots \ldots \ldots \ldots \ldots . \ldots \ldots 9$

II, $21 \ldots \ldots \ldots \ldots \ldots \ldots 346,364$

II $, 23,24 \ldots \ldots \ldots \ldots \ldots \ldots \ldots 356$

III, $10 \ldots \ldots \ldots \ldots \ldots \ldots \ldots \ldots \ldots$

III, $21 \ldots \ldots \ldots \ldots \ldots \ldots \ldots \ldots .420$

III, $30 \ldots \ldots \ldots \ldots \ldots \ldots .548,549$

Fons Vitae

I, 10, p. $13 \ldots \ldots \ldots \ldots . .599,601$

II, 1 , p. $21 \ldots \ldots \ldots \ldots \ldots .579$

II, 1, p. $24 \ldots \ldots \ldots \ldots \ldots . .599$

II, 14 , p. $47 \ldots \ldots \ldots \ldots .345,364$

II, 14 , p. $48 \ldots \ldots \ldots \ldots \ldots \ldots 6$

III, 27 , p. $143 \ldots \ldots \ldots \ldots \ldots 420$

III, 48 , p. $187 \ldots \ldots \ldots \ldots 548,549$

\section{Bahya Ibn Pakuda}

Hobot ha-Lebabot

Introduction . . . . . . . . . . . . .320

I, $5 \ldots \ldots 324,347,419,491,492$

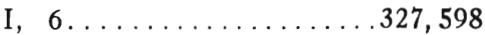

$\mathrm{I}, \quad 7 \ldots \ldots \ldots \ldots \ldots \ldots \ldots \ldots \ldots$

I, $9 \ldots \ldots \ldots \ldots \ldots \ldots \ldots \ldots 1$

$\mathrm{I}, 10 \ldots \ldots \ldots \ldots \ldots \ldots \ldots \ldots 40 . \ldots$

Pseudo-Bahya

Kitab $M a^{\prime} a$ an $a l-N a f s$

ch. $2 \ldots \ldots \ldots \ldots \ldots \ldots \ldots 00 . \ldots$

ch. $16 \ldots \ldots \ldots \ldots \ldots \ldots 461$

\section{Abraham Bar Hiyya}

Hegyon ha-Nefesh

I, p. 2a . . . . . . . . . . . . 639

I, p. 5 a................ 324

Megillat ha-Megalleh

p. $6 \ldots \ldots \ldots \ldots \ldots \ldots 641,661$

p. $10 \ldots \ldots \ldots \ldots \ldots \ldots \ldots 68 \ldots \ldots$

'Olam Katan

Joseph Ibn Zaddik

I, 3, p. $11 \ldots \ldots \ldots \ldots \ldots \ldots 452$

I, 3, p. $13 \ldots \ldots \ldots \ldots 361,588$ 
I, 3, p. $15 \ldots \ldots \ldots 355,446,456$ III, p. $49 \ldots \ldots \ldots \ldots \ldots \ldots 376$

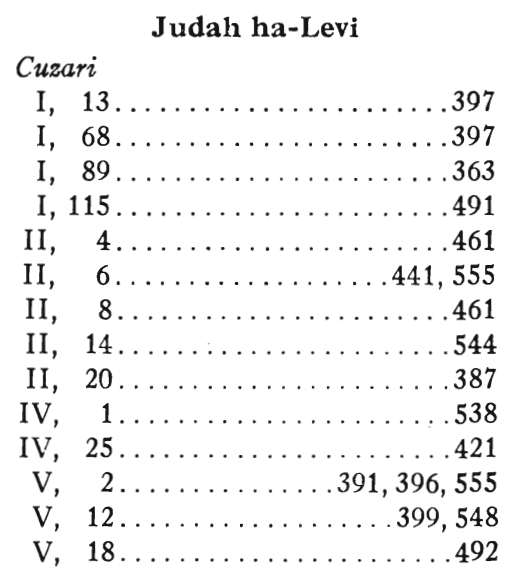

\section{Abraham Ibn Daud}

\section{Emunah Ramah}

I, $1 \ldots \ldots \ldots \ldots \ldots 418,689$

I, $2 . .570,572,577,578,580,598,599$

I, 2, p. $10 \ldots \ldots \ldots \ldots .579,587$

I, 2, p. $11 \ldots \ldots \ldots 573,574,576$, $579,588,590,600$

I, 3, p. $13 \ldots \ldots \ldots \ldots . .502,505$

I, 3, p. $14 \ldots \ldots \ldots \ldots 671,672$

I, $4 . .347,348,354,364,476,481,612$

I, 6, p. 28

I, 8, p. 41

.535

II, iv, 3, p. 63

.343

II, iv, 3, p. $64 \ldots \ldots \ldots \ldots 573,575$

II, iv, 3, pp. 64-65 ........575

II, iv, 3, p. $65 \ldots \ldots \ldots \ldots \ldots 390$

\section{Moses Maimonides}

\section{Moreh Nebukim}

Introduction . . . . . . . 28, 535

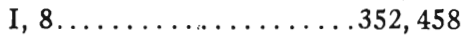

I, $10 \ldots \ldots \ldots \ldots \ldots \ldots \ldots 461$

I, $17 \ldots \ldots \ldots \ldots \ldots \ldots 72,700$

$\mathrm{I}, 19 \ldots \ldots \ldots \ldots \ldots 461,462$

I, $52 \ldots \ldots \ldots \ldots 636,687,688$

I, $56 \ldots \ldots \ldots \ldots \ldots \ldots \ldots . \ldots \ldots 34$

I, $58 \ldots \ldots \ldots \ldots \ldots \ldots 598,648$
$I, 64 \ldots \ldots \ldots \ldots \ldots \ldots \ldots 1$

I, $69 \ldots \ldots \ldots \ldots \ldots \ldots \ldots 5$

I, $71 \ldots \ldots \ldots \ldots \ldots \ldots \ldots \ldots \ldots$

I, $72 \ldots \ldots \ldots 55,606,607,700$

I, $73(2) \ldots \ldots \ldots \ldots \ldots 362,400$

I, $73(3) \ldots \ldots \ldots \ldots \ldots \ldots 454$

I, $73(8) \ldots \ldots \ldots \ldots \ldots \ldots .570$

I, $73(10) \ldots \ldots \ldots 343,465,466$

I, $73(11) \ldots \ldots \ldots \ldots .480,494$

I, $74(7) \ldots \ldots \ldots 485,491,570$

I, $76 \ldots \ldots \ldots \ldots \ldots \ldots 4$

II, Prop. IV . . . . . . . . 74, 399

II, Prop. XI ............. 577

II, Prop. XII............577

II, Prop. XVI . . . . . . . . . 577

II, Prop. XXI .......... 577

II, Prop. XXII ........... 577

II, Prop. XXV . . . . . . . . . .

II, $1 \ldots \ldots 122,324,329,422,550$, $559,605,606,611$

II, $4 \ldots \ldots 78,403,555,556,606$

II, $4-5 \ldots \ldots \ldots \ldots \ldots \ldots \ldots 535$

II, $12 \ldots \ldots 122,503,544,562,563$

II, $13 \ldots \ldots \ldots \ldots \ldots \ldots \ldots 68$

II, $13-27 \ldots \ldots \ldots \ldots \ldots \ldots 664$

II, $14 \ldots \ldots \ldots \ldots 325,401,679$

II, $16 \ldots \ldots \ldots \ldots \ldots \ldots \ldots . \ldots \ldots 1$

II, $18 \ldots \ldots \ldots \ldots \ldots \ldots \ldots 679$

II, $19 \ldots \ldots \ldots \ldots \ldots \ldots \ldots . \ldots \ldots 22$

II, $21 \ldots \ldots \ldots \ldots \ldots \ldots \ldots 68$

II, $22 \ldots \ldots \ldots \ldots \ldots \ldots \ldots .490$

II, $24 \ldots \ldots \ldots \ldots \ldots \ldots \ldots 452$

II, $27 \ldots \ldots \ldots \ldots \ldots \ldots \ldots 424$

II, $30 \ldots \ldots \ldots \ldots \ldots 63,664$

III, $7 \ldots \ldots \ldots \ldots \ldots \ldots \ldots 46$

III, $8 \ldots \ldots \ldots \ldots \ldots \ldots \ldots 696 \ldots . \ldots 69$

III, $10 \ldots \ldots \ldots \ldots \ldots \ldots \ldots 69$

III, $25 \ldots \ldots \ldots \ldots \ldots \ldots \ldots .324$

Millot ha-Higgayon

ch. $8 \ldots \ldots \ldots \ldots \ldots \ldots 326,397$

Kobez Teshubot ha-Rambam we-Iggerotaw

(a) Letter to Phinehas ben Meshullam:

I, p. $25 \mathrm{~b}$. 
(b) Letter to Samuel Ibn Tibbon:

II, p. 27 b . . . . . . . . 502, 636, 690 pp. 27b-28a . . . . . . . 694-5 p. $28 \mathrm{~b} \ldots \ldots \ldots \ldots \ldots \ldots . \ldots 321$ pp. $28 \mathrm{~b}-29 \mathrm{a}$. .322

Mishneh Torah, Sefer ha-Madda', Yesode ha-Torah

II, 3 .

III, 2

IV, 5 . $.400,450$

\section{Joseph Ibn 'Aknin}

Ma'amar. $587,590,592$

\section{Samuel Ibn Tibbon}

Ma'amar Yikkawu ha-Mayyim ch. 8, pp. $31-32 \ldots \ldots \ldots \ldots \ldots .460$

Bi' ur meha-Millot ha-Zorot. . . . . .564

\section{Joseph Zabara}

Sefer Sha'ashu'im

IX, 11 . $.567,568$

\section{David Kimhi}

On Isaiah 6, 3 459

\section{Azriel}

Perush 'Eser Sefirot (='Ezrat Adonai) p. 3b...............459, 460 p. $5 \mathrm{a} \ldots \ldots \ldots \ldots \ldots \ldots . . .459$

\section{Moses ha-Lavi}

* Ma'amar Elohi. .483

\section{Jacob Anatolio (?)}

Ruah Hen

ch. 11 503. 507

\section{Hillel of Verona}

Tagmule ha-Nefesh

I, 3, p. 3b...............501

I, 3, pp. 3b-4a...........501

I, 3, pp. 4b-5a...., ......598

\section{Commentary on Maimonides}

Twenty-five Propositions

Introduction . . . . . . . . . . 2, 319

Prop. IV...........521, 641, 672

Prop. V................ 522

Prop. VIII . . . . . . . . . . . . 553

Prop. XIII. . . . . . . . . . 501, 617

Prop. XIV .........399, 501, 628

Prop. XVIII . . . . . . . . . . . . . .676

Prop. XXII . . . . . . . . . . . . 687

Prop. XXIII . . . . . . . . . . 692, 696

Prop. XXIV............691, 697

Prop. XXV........ 575, 699, 700

\section{Shem-tob Falaquera}

Moreh ha-Moreh

I, $73 \ldots \ldots \ldots \ldots \ldots \ldots \ldots .417$

II, Prop. IV . . . . . . . . . . . . 503

II, Prop. V.............. 525

II, Prop. VIII...............553

II, Prop. XII...............682

II, Prop. XIV . . . . . . . . . . . . 630

II, Prop. XV . . . . . . . . . . . 646

II, Prop. XXII_........595, 596

II, $4 \ldots \ldots \ldots \ldots \ldots \ldots \ldots . \ldots . \ldots 57$

II $15 \ldots \ldots \ldots \ldots \ldots \ldots \ldots 417$

Reshit Hokmah

III, 1, p. $62 \ldots \ldots \ldots \ldots \ldots . .491$

\section{Isaac Ibn Latif}

Rab Pe'alim

$18 \ldots \ldots \ldots \ldots \ldots \ldots \ldots 6 . \ldots 62$

$60 \ldots \ldots \ldots \ldots \ldots \ldots \ldots \ldots+\ldots \ldots+\ldots \ldots$

$63 \ldots \ldots \ldots \ldots \ldots \ldots \ldots . \ldots 4$

Sha'ar ha-Shamayim...........538

Gershon ben Solomon

Sha'ar ha-Shamayim

I, $1 \ldots \ldots \ldots \ldots \ldots \ldots \ldots .413$

II, $3 \ldots \ldots \ldots \ldots \ldots \ldots \ldots 656$

III, $1 \ldots \ldots \ldots \ldots \ldots \ldots .567,568$

\section{Zerahia Gracian}

*Commentary on Maimonides'

Twenty-five Propositions........2 
Bahya ben Asher

On Exodus 34, $7 \ldots \ldots \ldots \ldots \ldots 460$

On Deuteronomy 3, 26.

460

\section{Joseph Caspi}

'Amude Kesef

p. 61 .

\section{Gersoniđes (Levi ben Gershon)}

Milhamot Adonai

I, 8 . .667

I, 9 547

III, 4 . 417

VI, i, 3 400

VI, i, 10 418

VI, i, $11 \ldots 348,352,387,464,477,478$

VI, i, 19 472

VI, i, 21 $421,638,652$

VI, i, 24 528

VI, i, 27 .424

* Commentary on Intermediate Physics III, ii, 5............527 III, iii, $4,2 \ldots \ldots 365,368,370,371$, $372,373,424,462,463$

IV, $i, 1,8 \ldots \ldots \ldots 360,361,442$

IV $, i, 1,9 \ldots \ldots \ldots \ldots \ldots 440$

IV, ii, $5 \ldots \ldots \ldots .342,406,410$

VIII, iv, $4 \ldots \ldots \ldots \ldots \ldots \ldots 56$

*Commentary on Epitome of Physics

III . . . . . . . . . . . . 430, 496

IV $\ldots \ldots \ldots \ldots \ldots \ldots \ldots \ldots 415$

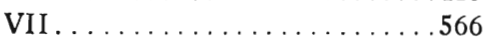

*Commentary on Intermediate De Caelo I, iv. . . . . . . . . . . . . . 556

I, vi.................6 633

*Commentary on Epitome of De Caelo I . . . . . . . . . . . . . . . .475

IV $\ldots \ldots \ldots \ldots \ldots \ldots \ldots \ldots 412$

Commentary on the Bible

Job, ch. 27
Moses ben Tobi

Al-Saba'niyyah 459,501

\section{Moses Narboni}

Commentary on Moreh Nebukim

I, $17 \ldots \ldots \ldots \ldots \ldots \ldots \ldots 0 . \ldots 70 \ldots$

I, $68 \ldots \ldots \ldots \ldots \ldots \ldots 60 \ldots \ldots$

I, $73(2) \ldots \ldots \ldots \ldots \ldots \ldots 416$

I, $73(3) \ldots \ldots \ldots \ldots \ldots 509,636$

I, $74(7) \ldots \ldots \ldots \ldots \ldots \ldots 487$

II, Prop. I . . . . . . . . . . 327

II, Prop. II . . . . . . . . . . 342

II, Prop. III . . . . . . . . . . . 492, 493

II, Prop. IV . . . . . . 498, 503, 507

II, Prop. V.............522

II, Prop. VI. . . . . . . . . . . 534

II, Prop. VII . . . . . . . . . . . 541

II, Prop. VIII . . . . . . . . . . 552

II, Prop. IX ............ . 561

II, Prop. XI . . . . . . . . 605, 608

II, Prop. XIV . . . . . . . . . . .628

II, Prop. XVIII . . . . . . . . .6676

II, Prop. XIX. . . . . . . . 682, 683

II, Prop. XXII . . . . . . . . . . . 686

II, Prop. XXIV . . . . . . . 510, 697

II, Prop. XXV . . . . . . . . 3, 672

* Commentary on Kawwanot ha-Pilosofim (Makaşid al-Falasifah)

II, Metaphysics.....4487, 583, 585, $586,590,665$

III, Physics.......362, 363, 409, $437,505,510,637,659$

* Commentary on Intermediate Physics

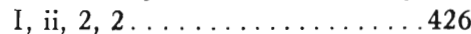

III, iii, $4,1 \ldots \ldots \ldots \ldots \ldots \ldots 33$

III, iii, $4,2 \ldots \ldots \ldots \ldots 424,429,478$

IV, ii, $5 \ldots \ldots \ldots \ldots \ldots \ldots \ldots 2$

* Commentary on Sermo de

Substantia Orbis........327, 585

\section{Isaac Albalag}

* Commentary on Kawwanot ha-Pilosofim (Makaşid al-Falasifah)

III . . . . . . . . . . 355, 400, 413 
Solomon Dapiera

Batte ha-Nefesh

p. 45 . .

p. 46 .

.501

Or Adonai

Hakdamah............23, 28

Haza'ah.................319

I, i, 31-32 ............ 324

I, ii, $19-20 \ldots \ldots \ldots \ldots \ldots . \ldots 324$

I, iii, $1 \ldots \ldots \ldots \ldots \ldots 319,398$

I, iii, $4 \ldots \ldots \ldots \ldots \ldots \ldots \ldots \ldots$

I, iii, $6 \ldots \ldots \ldots \ldots \ldots \ldots 320$

I, ii, $15 \ldots \ldots \ldots \ldots \ldots \ldots 612$

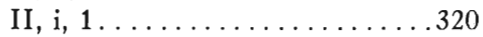

II, iii, $2 \ldots \ldots \ldots \ldots \ldots \ldots 612$

II, vi, $1 \ldots \ldots \ldots \ldots 486,550,667$

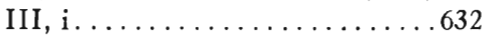

III, $i, 1 \ldots \ldots \ldots \ldots \ldots 320,424$

III, $i, 2 \ldots \ldots \ldots \ldots \ldots \ldots 664$

III, $i, 3 \ldots \ldots \ldots \ldots \ldots . . . .320,424$

III, $i, 4 \ldots \ldots \ldots \ldots 418,423,680$

III, $i, 5 \ldots \ldots \ldots \ldots \ldots 319,424$

III, ii, $2 \ldots \ldots \ldots \ldots \ldots \ldots 667$

III, viii, $2 \ldots \ldots \ldots \ldots \ldots \ldots 17$

IV, $2 \ldots \ldots \ldots 117,321,472,473$

IV, $3 \ldots \ldots \ldots \ldots \ldots \ldots 125,535$

IV, $4 \ldots \ldots \ldots \ldots \ldots \ldots \ldots \ldots$

Bittul 'Ikkere ha-Nozerim

p. $11 \ldots \ldots \ldots \ldots \ldots \ldots \ldots 29$

ch. 3, p. 27-28............ 29

ch. 3 , p. $30 \ldots \ldots \ldots \ldots \ldots \ldots \ldots$

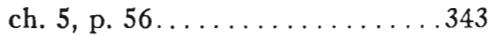

ch. 8 , p. $63 \ldots \ldots \ldots \ldots \ldots \ldots 17$

\section{Efodi}

Commentary on Moreh Nebukim

II, Prop. XIX. . . . . . . . . . 680 II, $12 \ldots \ldots \ldots \ldots \ldots \ldots \ldots 64$

Iggeret Al Tehi Ka-Aboteka. . . .343

'Ikkarim Joseph Albo

II, 11 .... .459
II, $17 \ldots \ldots \ldots .361,440,443,446$, $448,455,456,457$

II, $18 \ldots \ldots \ldots \ldots 422,656,658,663$

IV, $35 \ldots \ldots \ldots \ldots \ldots \ldots .568$

\section{Judah Messer Leon}

* Commentary on Intermediate

Categories

III, $2 \ldots \ldots \ldots \ldots \ldots \ldots \ldots . \ldots 6$

\section{Isaac ben Shem-tob Ibn Shem-tob}

* First Commentary on Intermediate

Physics

I, ii, 2, 2 . .428

III, iii, $4,1 \ldots \ldots \ldots \ldots \ldots . .396$

III, iii, $4,2 \ldots \ldots \ldots \ldots \ldots 425,431$

IV, $i, 1,9 \ldots \ldots \ldots \ldots \ldots .440$

IV, ii, $5 \ldots \ldots \ldots \ldots \ldots \ldots \ldots$

IV, iii, $4 \ldots \ldots \ldots \ldots \ldots \ldots .418$

*Second Commentary on Intermediate Physics

I, ii, $2,2 \ldots \ldots \ldots \ldots \ldots \ldots .428$

III, iii, $4,1 \ldots \ldots \ldots \ldots 395,426$

III, iii, $4,2 \ldots \ldots \ldots \ldots 424,479$

IV, ii, $5 \ldots \ldots \ldots \ldots \ldots . . . \ldots 343$

IV, iii, $4 \ldots \ldots \ldots \ldots \ldots . .650$

*Third Commentary on Intermediate Physics

IV, ii, $5 \ldots \ldots \ldots \ldots \ldots . \ldots 343,398$

\section{Shem-țob ben Joseph} Ibn Shem-tob

Commentary on Moreh Nebukim

I, $1 \ldots \ldots \ldots \ldots \ldots \ldots$.........607

I, $17 \ldots \ldots \ldots \ldots \ldots \ldots$. 700

I, $68 \ldots \ldots \ldots \ldots \ldots \ldots 60.606$

I, $72 \ldots \ldots \ldots \ldots \ldots \ldots 606$

I, $73(10) \ldots \ldots \ldots \ldots \ldots \ldots 343$

II, Prop. I . . . . . . . . . . . . 33

II, Prop. IX............ 562

II, Prop. XI...............604

II, Prop. XIV . . . . . . . . . .628

II, Prop. XVII . . . 323, 413, 673, 675

II, Prop. XXII . . . . . . . . . 598 
II, 1

II, 14 $\ldots 325$

* Commentary on Intermediate Physics

I, ii, 2, 2.......... 33,427

III, iii, $4,1 \ldots \ldots \ldots \ldots \ldots . \ldots 33,394$ IV $, \mathrm{i}, 8 \ldots \ldots \ldots \ldots \ldots \ldots 41$

Neveh Shalom

\section{Abraham Shalom}

$\mathrm{V}, 11$, p. $81 \mathrm{~b}$

VII, i, 3

VII, i, 3, p. $100 \mathrm{~b}$

VIII, 3, p. $125 \mathrm{~b}$

VIII, 9, p. $144 \mathrm{~b}$

XII, i, 3, p. 304a .649

\section{Isaac Arama}

'Akedat Yizhak

Sha'ar II... .538

\section{Menahem Bonafos}

\section{Sefer ha-Gedarim}

p. $19 \mathrm{a}$

p. $61 \mathrm{~b}$

\begin{tabular}{|c|c|}
\hline & Isaac Abravanel \\
\hline Mif 'alo & t Elohim \\
\hline I, $3 \ldots$ & $\ldots \ldots \ldots \ldots 539$ \\
\hline II, $3 \ldots$ & $\ldots \ldots \ldots 597,682$ \\
\hline IX, $4 \ldots$ & $\ldots 481,496$ \\
\hline IX, $7 \ldots$ & $\ldots \ldots \ldots \ldots \ldots \ldots \ldots 17$ \\
\hline
\end{tabular}

Shamayim Hadashim

I . . . . . . . . . . . . . . . . . 323

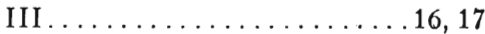

She'elot Saul

pp. 9b-10b. . . . . . . . . . . . . .589

p. $18 \mathrm{ff} . \ldots \ldots \ldots \ldots \ldots \ldots \ldots 58$

p. $18 \mathrm{a}-\mathrm{b} \ldots \ldots \ldots \ldots \ldots \ldots \ldots \ldots . . .580$

p. 18 b.... $578,584,585,587,590$

p. $19 \mathrm{~b}-20 \mathrm{a} \ldots \ldots \ldots \ldots \ldots \ldots 59$

p. $20 \mathrm{~b} \ldots \ldots \ldots \ldots \ldots \ldots \ldots 60 \ldots \ldots$

\section{Asher Crescas}

Commentary on Moreh Nebukim

II, Prop. XIX...........680

Judal Aryeh Moscato

Commentary Kol Yehudah on Cuzari

IV, 1 .538

\section{Gedaliah Lippschitz}

Anafim and Shorashim on 'I kkarim

II, $17 \ldots \ldots \ldots \ldots \ldots \ldots 361,457$

\section{Baruch Spinoza}

Ethics

I, Prop. XV, Schol...........36

Epistolae

$X I I(X X I X) \ldots \ldots \ldots \ldots \ldots \ldots \ldots$

\section{EUROPEAN AUTHORS}

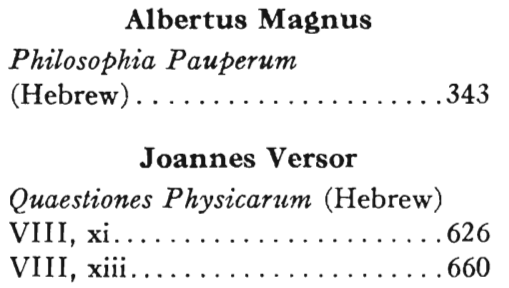

Giovanni Francesco

Pico della Mirandola

Examen Doctrinae Vanitatis Gentium VI, $2 \ldots \ldots \ldots \ldots 625,626,560$
VI, $3 \ldots \ldots 650,658,661,662,663$

VI, $4 \ldots \ldots \ldots \ldots \ldots \ldots 49,456$

VI, $6 \ldots \ldots 398,403,412,414,417$, $418,419,422$

VI, 18 412,564

\section{Giordano Bruno}

De l'Infinito Universo et Mondi

I, p. $309 \ldots \ldots \ldots \ldots \ldots \ldots \ldots 43$

I, p. $310 \ldots \ldots \ldots \ldots \ldots \ldots 422$

II, p. $318 \ldots \ldots \ldots \ldots \ldots \ldots 613$

II, p. $326 \ldots \ldots \ldots \ldots \ldots 464,470$

II, p. $328 \ldots \ldots \ldots \ldots \ldots 431,472$ 
II, p. 340 .

466

III, p. 356

.414

IV, p. 365 476

IV, p. 369 . 472

De Immenso et Innumerabilibus

I, vi..................443

II, ii . . . . . . . . . . . . . 464

II, iv .................431

II, vii. . . 466

II, $\mathbf{x}$...... 470
De la Causa, Principio, et Uno

II, p. $240 \mathrm{f} . \ldots \ldots \ldots \ldots \ldots .560$

Locke

An Essay concerning Human Understanding

IV, xviii, \$21 .............326

\section{Leibnitz}

Nouveaux Essais

II, xiii, $\$ 17 \ldots \ldots \ldots \ldots \ldots . \ldots 123$ 